\title{
Conditional Diagnosability Algorithm for the Exchanged Hypercube under the PMC model
}

\author{
Weixia Gui ${ }^{1,2, a}{ }^{*}$, Jiarong Liang ${ }^{1,2, b}$, Cui Liü, \\ ${ }^{1}$ College of Automation Science and Engineering, South China University of Technology, \\ Guangzhou 510641, China \\ ${ }^{2}$ College of Computer and Electronic Information, Guangxi University, Nanning 530004, China \\ wxgui@126.com, ’bxuliangjr@163.com, ${ }^{c} 1978037519 @ q q . c o m$
}

Keywords: The Exchanged Hypercube; Conditional Diagnosability; PMC model; Diagnosability
Algorithm

Abstract. System-level fault identification is a key subject for maintaining the reliability of multiprocessor interconnection network. As an important interconnection network, the Exchanged Hypercube $E H(s, t)$ not only kept numerous desirable properties of the hypercube, but also reduced the interconnection complexity. This paper present one fast and accurate conditional diagnosability algorithm for $E H(s, t)$ to identify the faulty vertices under the PMC model. The time complexity of the algorithm is $O\left(N^{2}\right)$ for the $E H(s, t)(t \geq s \geq 3)$ with $N$ vertices.

\section{Introduction}

As the semiconductor technology continuously advances, the application of large-scale multiprocessor computer systems is more widely. As we know, the systems can contain hundreds of thousands of processors that communicate by exchanging messages through an interconnection network. It's inevitable that some processors may fail in there. Before being repaired, all the fault processors in the system must be identified. The problem of fault self-diagnosis in multiprocessor systems has gained increasing importance.

Several famous different strategies have been developed for self-diagnosis in multiprocessor systems. Preparata et al. [1] first proposed a model called PMC model. Under this model, processors test each other if they are adjacent directly. It assumes that the tests performed by fault-free vertices are always correct, whereas tests performed by faulty vertices are unreliable. Preparata et al. [1] defined the notion of $t_{p}$-diagnosable system in which all the faulty processors can be identified provided the number of faulty processors does not exceed $t_{p}$. The maximum number $t_{p}$ such that the system is $t_{p}$-diagnosable is called $t_{p}$-diagnosability. In order to improve the capability of self-diagnosis, Friedman and Kavianpour[2,3] introduced the concept of $t_{1} / t_{1}$-diagnosable system in which all the faults can be isolated to within a set of at most $t_{1}$ vertices provided the number of faulty vertices does not exceed $t_{1}$. The maximum number $t_{1}$ such that the system is $t_{1} / t_{1}$-diagnosable is called $t_{1} / t_{1}$-diagnosability (or pessimistic diagnosability). Yang et al. [4] proved that at most one fault-free vertex may be identified as faulty in $t_{1} / t_{1}$-diagnosable system. Also based on the unlikelihood of failure of all neighbors of any particular vertex, Lai et al. [5] proposed the definition of the conditional diagnosability of interconnection networks. Given an interconnection network $G, G$ is called conditionally $t$-diagnosable if and only if any two conditional sets $F_{1}, F_{2}$, such that $\left|F_{1}\right|,\left|F_{2}\right| \leq t$, are distinguishable. The conditional diagnosability of an interconnection network $G$ is the maximum integer $t$ such that $G$ is conditionally $t$-diagnosable.

As one of important applications of a parallel computer network model, hypercube's topological properties, routing algorithms, fault tolerance, and embedding have aroused the interests of many experts and scholars, and then have got a lot of valuable results[6-8]. However, hypercube scales too rapidly as $n$ increases. Existing research has proposed some networks that are variations of the hypercube network variants: folded hypercube, twisted cube network, cross cube network and so on. The Exchanged Hypercube [9] is a new variant of the hypercube; it not only keeps the several desirable 
properties of the hypercube such as low diameter, bipancyclicity, and super connectivity but also reduces the complexity of the network interconnection. Some research showed the exchanged hypercube has nice recursiveness and preferable network parameters [10-12].It has received a great deal of attentions and researches. The connectivity and conditional connectivity of $E H(s, t)$ are determined in [12-14]. The topological properties and embedding issues of $E H(s, t)$ are investigated in [15]. However,a conditional diagnosability algorithm for the exchanged hypercube has not been studied yet.

The rest of this paper is organized as follow: in the next section, some fundamental definitions and notions are introduced, main results are proved and an algorithm is presented in Section 3. The last section concludes the paper and draws some comments about ongoing work.

\section{Preliminaries}

An interconnection network can be modeled by an undirected graph $G=(V, E)$, where $V$ is the set of processors and $E$ is the set of communication links. For graph terminology and notation not defined here we follow [16]. The neighbor-set of $V^{\prime}$ in $G$ is defined as $\Gamma\left(G, V^{\prime} \quad\right)=\{v \in V(G) \mid(u, v) \in E(G), u$ $\left.\in V^{\prime}, V^{\prime} \square V(G)\right\}-V^{\prime} . N(u)=\{v \in V(G) \mid v$ is adjacent to $u\}$.

Definition 1.[9]The Exchanged Hypercube is defined as an undirected graph: $E H(s, t)=(V, E)(s \geq 1, t$ $\geq 1), V$ is the set of vertices and $V=\left\{a_{s-1} \ldots a_{0} b_{t-1} \ldots b_{0} c \mid a_{i}, b_{j}, c \in(0,1), i \in[0, s), j \in[0, t)\right\}$; and $E$ is the set of edges:(1) $\left(v_{1}, v_{2}\right) \in V \times V$ if $v_{1} \oplus v_{2}=1$; (2) $v_{1}[s+t, t+1]=v_{2}[s+t, t+1], H\left(v_{1}[t, 1], v_{2}[t, 1]\right)=1$ if $v_{1}[0]=v_{2}[0]=1 ;(3) v_{1}[t, 1]=v_{2}[t, 1], H\left(v_{1}[s+t, t+1], v_{2}[s+t, t+1]\right)=1$ if $v_{1}[0]=v_{2}[0]=0$, where $\oplus$ denotes the exclusive-OR operator, $v[x, y]$ denotes the bit pattern of $v$ between dimensions $x$ and $y$ inclusive. $H\left(v_{1}\right.$, $\left.v_{2}\right)$ denotes the Hamming distance between vertices $v_{1}$ and $v_{2}$, where $\left(v_{1}, v_{2}\right) \in V \times V$.

Fig.1 shows an exchanged hypercube when $s=1, t=2$.

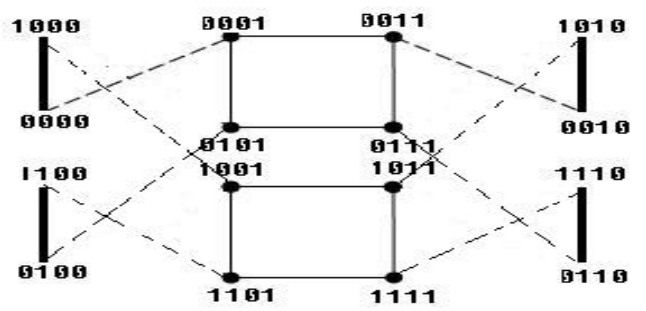

Fig.1. An Exchanged Hypercube, $E H(1,2)$

\section{Main Results}

Theorem 1. [17] The conditional diagnosability of an Exchanged Hypercube under the PMC model is $t_{c}($ $E H(s, t))=4 s-3$ when $t \geq s \geq 3$.

Definition 2.If all the processors in the set are faulty, the set is called a faulty set denoted by $F$, otherwise, if all the processors in the set are faulty free, the set is called a faulty free set denoted by $T$. Theorem 2. [18] If one vertices set $M$ can be derived from conditional diagnosed under the PMC model, the vertices must be connected in $M$, and $M$ must be either faulty set or faulty free set.

Theorem 3. [18] If one vertices set $M$ can be derived from conditional diagnosed under the PMC model, and $M$ has only one vertex, $M$ must be a faulty set. If $M$ doesn't have neighbor vertices set, $M$ must be a faulty free set.

Theorem 4. [18] If $M$ is a faulty set, it must be neighbor with at least one faulty free set, and all the sets of neighboring vertices with the faulty free sets must be faulty sets.

Theorem 5. EH $(s, t)(t \geq s \geq 3)$ has at most 3 faulty free sets, there must be two faulty free sets, each set contains two elements. If $E H(s, t)$ only has two faulty free sets, the number of elements in one of sets is no more than 5 .

Proof: At first we prove that $E H(s, t)(t \geq s \geq 3)$ has at most 3 faulty free sets, there must be two faulty 
free sets, each set contains two elements. By the definition of conditional diagnose, the number of vertices in each faulty free set is not less than 2.Assume that $E H(s, t)$ has 3 faulty free sets, and two faulty free sets contain more than two elements, one faulty free set contains three elements. As three elements are in two dimensions, we delete the remaining elements in $(s-2)$ dimensions for isolating the three vertices. Additional, the two dimensions including three elements need delete one element too, that is the number of elements in vertex cut is $3 \times(s-2)+1$, and is $s+2$ less than the maximum number of fault vertices $(4 s-3)$, but $s+2$ vertices can't cut two vertices. So the number of elements in the two faulty free sets is 2 .

And then we prove that if $E H(s, t)$ only has two faulty free sets, the number of elements in one of sets is no more than 5.Suppose that the number of elements in one of sets is 6.So the vertex cut departing 6 elements is at least $6 \times(s-3)+2$, but it is more than the maximum number of fault vertices, a contradiction. Hence the number of elements in one of fault free sets is no more than 5.

By the Theorem 1, the conditional diagnosability of an Exchanged Hypercube under the PMC model is no more than $4 s-3$. We present a conditional diagnosability algorithm which can start from any vertex, traverse the whole interconnection network and classify all the vertices, put the faulty vertices into faulty set and put the faulty free vertices into faulty free set respectively. And then, the algorithm identifies the remaining vertices by Theorem $2 \sim$ Theorem5.The algorithm is described as follows in detail, a faulty free set denoted by $T$, and $T=\left\{t_{i} \in \mathrm{V} \mid t_{i}\right.$ is a faulty free vertex $\}$, a faulty set is denoted by $F$, and $F=\left\{f_{i} \in \mathrm{V} \mid f_{i}\right.$ is a faulty vertex $\}$, the set including vertices which can't be identified is denoted by $M$, the neighbor-net of $u$ is denoted by $N(u), \sigma(u, v)$ is the result of vertex $u$ test vertex $v$, the input of the algorithm is $E H(s, t)$, where $t \geq s \geq 3$, the number of faulty vertices is $N \leq 4 s-3$.

\section{Algorithm 1}

Step 1: Initialization. Let any not diagnosed vertex $u$ become the beginning, $k=1, S=0, i=1, M_{i}(0)=u$ Step 2: According the PMC model, the algorithm uses Breadth-First-Search starting from the vertex $u$, traverses and finds the neighboring vertex which is not diagnosed by the order from low dimension to high dimension. If the algorithm doesn't search such vertices, then goes to step 4; otherwise $N(u)=v$, then goes to step 3

Step 3: Diagnose vertex $u$ and its neighbor vertex $v$.

If $u$ and $v$ are satisfied $\sigma(u, v)=0$, then

If $\sigma(v, u)=0$ then $M_{i}(k)=v, u=v, k=k+1$, the algorithm continues step 2;

If $\sigma(v, u)=1$, then vertex $u$ is faulty, $M_{i}$ is a faulty set. The elements in $M_{i}$ are put into the faulty set $F$, at the same time, let $v$ become the start vertex in $M_{i}$, that is $M_{i}(0)=v, u=v$, then goes to step 2;

If the neighbor vertex $v$ of the $i$ th dimension is satisfied $\sigma(u, v)=1$, then $v \notin M_{i}$, goes to step 2 .

Step 4: Search for a vertex $s$ which is not included in any sets, that is $s \notin\left\{M_{1} \cup M_{2} \cup \ldots M_{i} \cup F\right\}$, there exists vertex $s$, then $u=s$, goes to step 2,otherwise, goes to step 5

Step 5: We obtain some vertices sets such as $M_{1}, M_{2} \ldots, M_{i}$ and $F$. By the theorem 3 theorem 5, we can identify $M_{i}$ is a faulty set or a faulty free set, and then the elements of $M_{i}$ can be put into $F$ or $T$.

Step 6: All sets $T$ which obtain form the above steps are the faulty free sets of $E H(s, t)$, and all sets $F$ are the faulty sets of $E H(s, t)$.

Algorithm analyses: By the Theorem 1, the conditional diagnosability of $E H(s, t)(t \geq s \geq 3)$ under the PMC model is no more than $4 s-3$. The main thought of this algorithm starts from any vertex, classifies all the vertices in the $E H(s, t)$, then puts the vertices identified as faulty (or faulty free) into faulty sets (or faulty free sets). Those vertices that don't be identified are included in many sets $M_{1}, M_{2} \ldots, M_{i}$. By the theorem 3 theorem 5, we can judge $M_{i}$ is a faulty set or a faulty free set, then the 
elements of $M_{i}$ can be put into $F$ or $T$.

The following analyze the algorithm's Time Complexity. The worst cases of Step 2 and Step 3 traverse in the whole $E H(s, t)$ through Breadth-First-Search. $E H(s, t)$ use adjacency list as store structure, the time complexity is $O\left(N^{2}\right)$, and the time complexity of step 4 is $O(1)$, the same as step 5 . Hence, the Time Complexity of the algorithm is $O\left(N^{2}\right)$ with $N$ vertices.

\section{Conclusions}

The fault tolerance of interconnection network with respect to their self-diagnosis capabilities is a very interesting research field, in which many problems remain open. In this paper, under the PMC model we proposed an effective and efficient conditional diagnosability algorithm for the Exchanged Hypercube, in which some links are reduced systematically. The algorithm traverses the whole $E H(s, t)$ by Breadth-First-Search and classifies all the vertices. The main contribution of this paper is to provide a fast and accurate way to identify faulty vertices in $E H(s, t)$. There are several different fault diagnosis models in the area of diagnosability. It is worth investigating, under various models, the faster and more accurater algorithm for $E H(s, t)$, even for other excellent interconnection networks.

Acknowledgment. This work is supported by National Natural Science Foundation of China (Grant No. 61363002), Program for New Century Excellent Talents in University of China" (NCET-06-0756) and Education Department of Guangxi under the grant(No. LX2014002). The authors also gratefully acknowledge the helpful comments and suggestions of the reviewers, which have improved the presentation.

\section{References}

[1] F. P. Preparata, G. Metze, R. T. Chien, On the connection assignment problem of diagnosable systems. IEEE Transactions on Electronic Computers, 16 (1967) 848-854.

[2]A. Kavianpour, A. D. Friedman, Efficient design of easily diagnosable systems. in Proc. 3rd USA-Japan Computer Conf,(1978) 251-257.

[3] A. D. Friedman,A new measure of digital system diagnosis. Digest of the International Sympsium on Fault Tolerant Computing, (1975) 167-170.

[4]C. L. Yang, G. M. Masson, R. A. Leonetti. On fault isolation and identification in $\mathrm{t}_{1} / \mathrm{t}_{1}$-diagnosable systems. IEEE Transactions on Computers, 100(1986) 639-643.

[5]P.-L. Lai, J. J. Tan, C.-P. Chang, and L.-H. Hsu, Conditional diagnosability measures for large multiprocessor systems, IEEE Transactions on Computers, 54(2005) 165 -175.

[6]K. Day, A. Tripathi, A comparative study of toplogical properties of hypercubes and star graphs, IEEE Transactions on Parallel and Distributed Systems, 5(1994)31-38.

[7]W.H. Yang, J.X. Meng, Generalized measures of fault tolerance in hypercube network, Applied Mathematics Letters, 25(2012)1335-1339.

[8]S.A. Choudum, S. Lavanya, Embedding a subclass of trees into hypercubes, Discrete Mathematics, 311(2011)866-871.

[9]P.K.K. Loh, W.J. Hsu and Y. Pan, The exchanged hypercube, IEEE Transactions on Parallel and Distributed Systems, 16(2005)866-874

[10]S. Klavžar, M.J. Ma, The domination number of exchanged hypercubes, Information Processing Letters, 114(2014)59-162.

[11T.H. Tsai, Y.C. Chen, J. M. Tan, Internally disjoint paths in a variant of the hypercube, Advances in Intelligent Systems and Applications, 20(2013)89-96.

[12]X.J. Li, J.M. Xu, Generalized measures of fault tolerance in exchanged hypercubes, Information Processing Letters, 113(2013)533-537.

[13] M. Ma. The connectivity of exchanged hypercubes. Discrete Mathematics Algorithms and Applications, 2(2010) 213-220.

[14] M. Ma, L. Zhu. The super connectivity of exchanged hyper-cubes. Information Processing Letters, 111(2011) 360-364. 
[15] X. Y. Wang, J. R. Liang, Q. L. Dou. Research on topological properties and embedding issues of the exchanged hypercube. Acta Electronica Sinica,40(2012) 669-673.

[16] R. J. Trudeau. Introduction to graph theory. Courier Dover Publications, 2013.

[17] Xiao-xue Yang,Li-mei Lin, Shu-ming Zhou, Conditional diagnosability of exchanged hypercube under PMC model, Journal of Shandong University (Natural Science), 47(2012)45-50.(in Chinese) [18] Liguo Zhang,Huimin Du , Jungang Han,Conditional diagnosability algotithm for hypercube under the PMC model. Journal of xidian University, 39(2012)148-153.(in Chinese) 